\title{
A Clinical Case of 5 Times \\ Irradiated Recurrent Orbital Hemangiopericytoma
}

\author{
Konstantin Gordon ${ }^{a} \quad$ Igor Gulidov ${ }^{a}$ Danil Gogolina ${ }^{a}$ Olga Lepilina ${ }^{b}$ \\ Olga Golovanova $^{c}$ Alexey Semenova Sergey Dujenko ${ }^{c}$ \\ Kira Medvedeva $^{a} \quad$ Sergey Koryakin $^{c}$ Sergey Ivanov ${ }^{d} \quad$ Andrey Kaprin ${ }^{e}$ \\ aDepartment of Proton Therapy, A. Tsyb Medical Radiological Research Center, Obninsk, \\ Russia; ${ }^{b}$ Department of Dosimetry, A. Tsyb Medical Radiological Research Center, Obninsk, \\ Russia; 'Department of Radiophysics, A. Tsyb Medical Radiological Research Center, \\ Obninsk, Russia; ${ }^{\mathrm{d} A}$. Tsyb Medical Radiological Research Center, Obninsk, Russia; ${ }^{\text {eNational }}$ \\ Medical Research Radiological Center, Moscow, Russia
}

\author{
Keywords \\ Proton therapy $\cdot$ Re-irradiation $\cdot$ Hemangiopericytoma
}

\section{Abstract}

Orbital hemangiopericytomas (HPCs) are rare mesenchymal tumors with a high tendency to recur. Treatment options are quite limited in case of a relapse, but re-irradiation can be useful. Most of the available data limit the possibility of re-irradiation, while novel techniques (e.g., pencil beam proton therapy [PT]) open new approaches for the safe repeating of treatment. To the best of our knowledge, this is the first well-documented case of multi-times $(>3)$ irradiation of tumors located intracranially. The case reported here describes orbital HPCs with proton irradiation performed two times since 1999 in a 30-year-old woman with a medical history as well as surgery followed by conventional radiotherapy (RT) and chemotherapy, and two times stereotactic RT (in 2009 and 2013).In 2016 the patient came to our hospital with the 3 rd relapse of the tumor, located in the left orbit, with an intracranial part, involving cavernous sinus, which was close to the temporal lobe. The 4th course of irradiation was done in May to June 2016 by pencil beam PT. Radiation necrosis occurred after 2 years and was treated with bevacizumab (BVZ). Three years later, another relapse was treated with PT and BVZ. The 9 -month follow-up showed complete tumor response without signs of brain toxicity. The patient died due to a brain abscess 1 year after the 5 th irradiation. This case shows a possibility of irradiation, applied 5 times to the same location, with promising results and manageable toxicity. 


\section{Introduction}

First described in 1923 [1] by Zimmermann, hemangiopericytoma (HPC) is a rare mesenchymal tumor originating from uncontrolled growth of pericytes. Despite the rarity of HPCs (around 1\% of all vascular tumors), the orbit location is rarer for this type of tumor, which corresponds to $0.8-3 \%$ of all primary orbital tumors [2]. The current standard of care for HPCs has not dramatically changed since the early 1970s with the importance of maximum tumor excision [3]. The role and the optimal dosing of radiotherapy (RT) for orbital HPCs are still under discussion. Most current publications present case reports of combined treatment, while the possibility of complete resection in this location is rare. Tijl et al. [4] reported the use of RT postoperatively. Setzkorn et al. [5] described treatment for recurrent HPC of the orbit with postoperative RT to a dose of $64.8 \mathrm{~Gy}$. Shinder et al. [6] presented a case of preoperative use of RT in recurrent orbital HPC.

These tumors have a high tendency for recurrence, but the treatment options are usually quite limited. Due to the long period before relapse, repeated RT can be one of them.

Cases of re-irradiation of brain tumors are rarely presented. The high risk of radiationinduced brain tissue necrosis still prevails in the professional society [7]. Nevertheless, in the last decade, we have seen an increasing interest in re-irradiation, especially for head and neck and brain location. Novel precise radiation techniques [8-10] and anti-necrotic drug treatment [11] help release re-irradiation safely. Besides, multiple repeated RT is an occasionally used option.

\section{Case Report}

In 2016, a 30-year-old woman with a 3rd relapse of the left orbital HPC was presented to our Tumor Board. She had received her first treatment from November 1999 to March 2001, which was incomplete surgical resection of the tumor, histologically reported as a benign HPC. Surgery was followed by RT, 60.0 Gy in $30 \mathrm{Fx}$.

In 2009 the tumor recurred in the same place, and the patient was treated with Cyberknife SRT, 24 Gy in 3 Fx. After 6 months, the patient lost the vision in her left eye, though it was a complete response. In 2013 another recurrence occurred. The patient again received SRT with 30 Gy in 6 Fx.

In April 2016, the patient came to our hospital with a local unresectable 3rd tumor relapse (shown in Fig. 1A).

The treatment options were limited due to the unresectability and benign nature of the tumor. Because no MRI indications of radiation brain tissue necrosis were reported and there was high conformity with previous stereotactic RT, long-term disease history, and gaps between RT courses, we decided to irradiate the patient again. The case was discussed at the Tumor Board, and the Institutional Ethical Committee approved the treatment strategy.

The 4th course of irradiation was done in May to June 2016. A dose of 60 Gy with $2.0 \mathrm{~Gy} /$ Fx was delivered via fixed horizontal spot-scanning proton therapy (PT) in a seated position supported with image guidance with built-in CBCT [12]. Standard immobilization equipment (thermoplastic mask) was used to ensure the reproduction of the patient position.

Maximum high conformity and temporal lobe sparing were chosen as the main goals of treatment planning. The patient was blind in her left eye, so we did not need to spare the left optical nerve, which was wholly involved in the tumor, but the other goal was to reduce the dose to the chiasma and contralateral optical nerve as much as possible. GTV was delineated based on MRI and CT co-registration. The planning volume was done by adding a 3-mm margin to GTV. The GTV volume was $8.2 \mathrm{~cm}^{3}$, and the final PTV was $19.4 \mathrm{~cm}^{3}$.

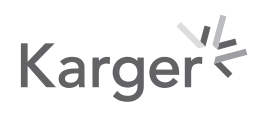



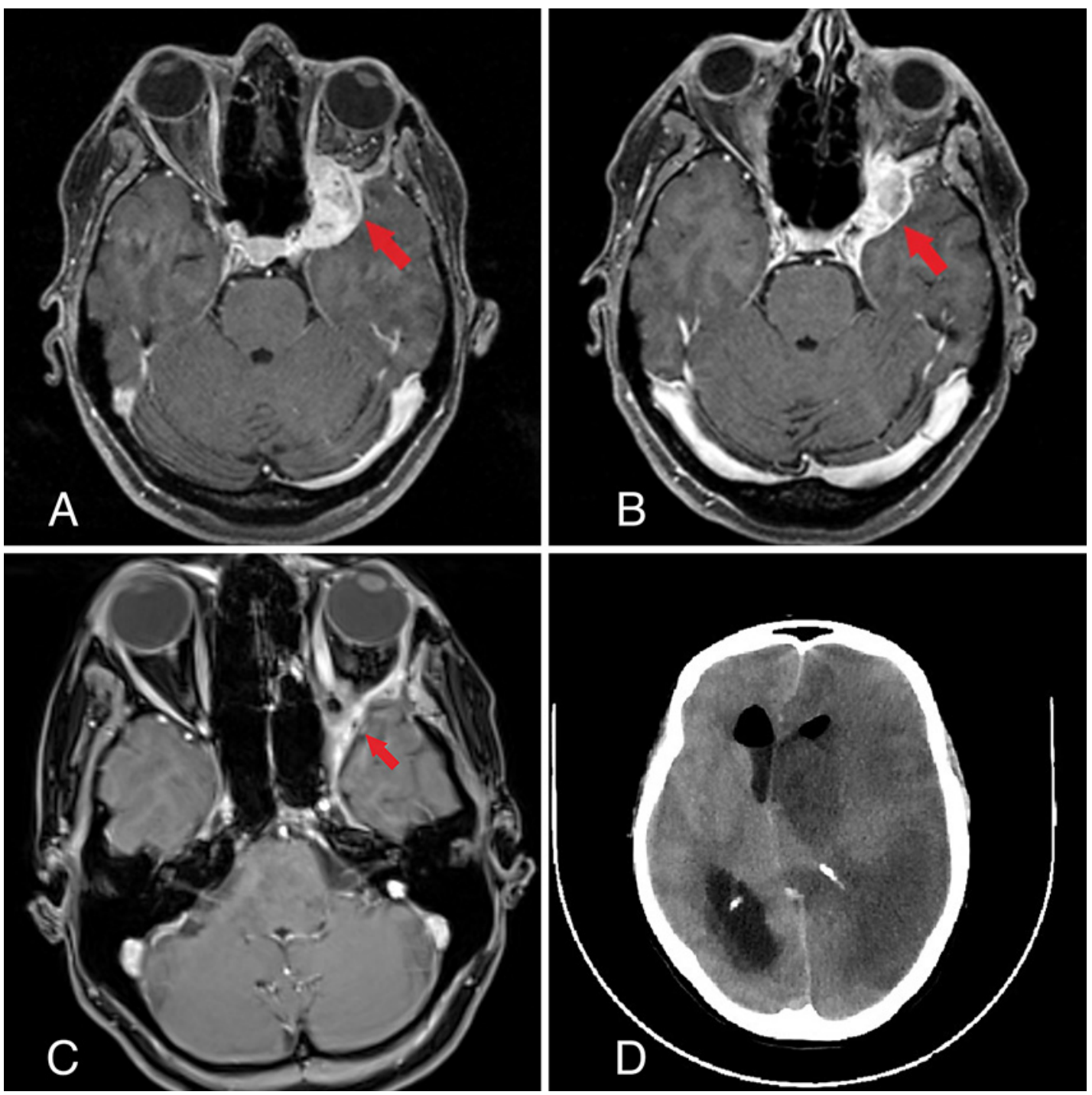

Fig. 1. Diagnostic imaging during the patient's follow-up since 2016.

The maximum dose to the PTV was $112.9 \%$, and the minimum dose was $75.9 \%$. The $\mathrm{V}_{95}$ (volume receiving $95 \%$ of the prescribed dose) was $92.4 \%$, and $\mathrm{D}_{95}$ (dose to $95 \%$ of volume) was 56.3 GyRBE (relative biological efficacy $=1.1$ ). The dose coverage was compromised to spare the temporal lobe. The right eyeball and right lens received 0 GyRBE. The dose to the left temporal lobe was 56.5 GyRBE (maximum dose), and to the right temporal lobe, the dose was $0 \mathrm{~Gy}$ (maximum dose). The maximum brainstem dose was $16.1 \mathrm{GyRBE}$, the maximum chiasma dose was 29.8 GyRBE, and the maximum dose to the right optical nerve was 7.8 GyRBE. The mean dose to the hypophysis was 3.0 GyRBE, and the midbrain dose was 1.0 GyRBE. We made a two-field plan with a perpendicular setup as the shortest way to the tumor. The PT dose distribution is shown in Figure 2A.

The partial response (tumor shrinkage of $79.3 \%$, from 8.2 to $1.7 \mathrm{~cm}^{3}$ ) was registered after 12 months. After 24 months, the next MRI reported a small $\left(2.8 \mathrm{~cm}^{3}\right)$ lesion in front of the left temporal lobe with peripheral contrast enhancement, suspected to be a radiation injury. ${ }^{18} \mathrm{~F}$-tyrosine PET/CT confirmed the adverse event. A combination of steroids and bevaci- 

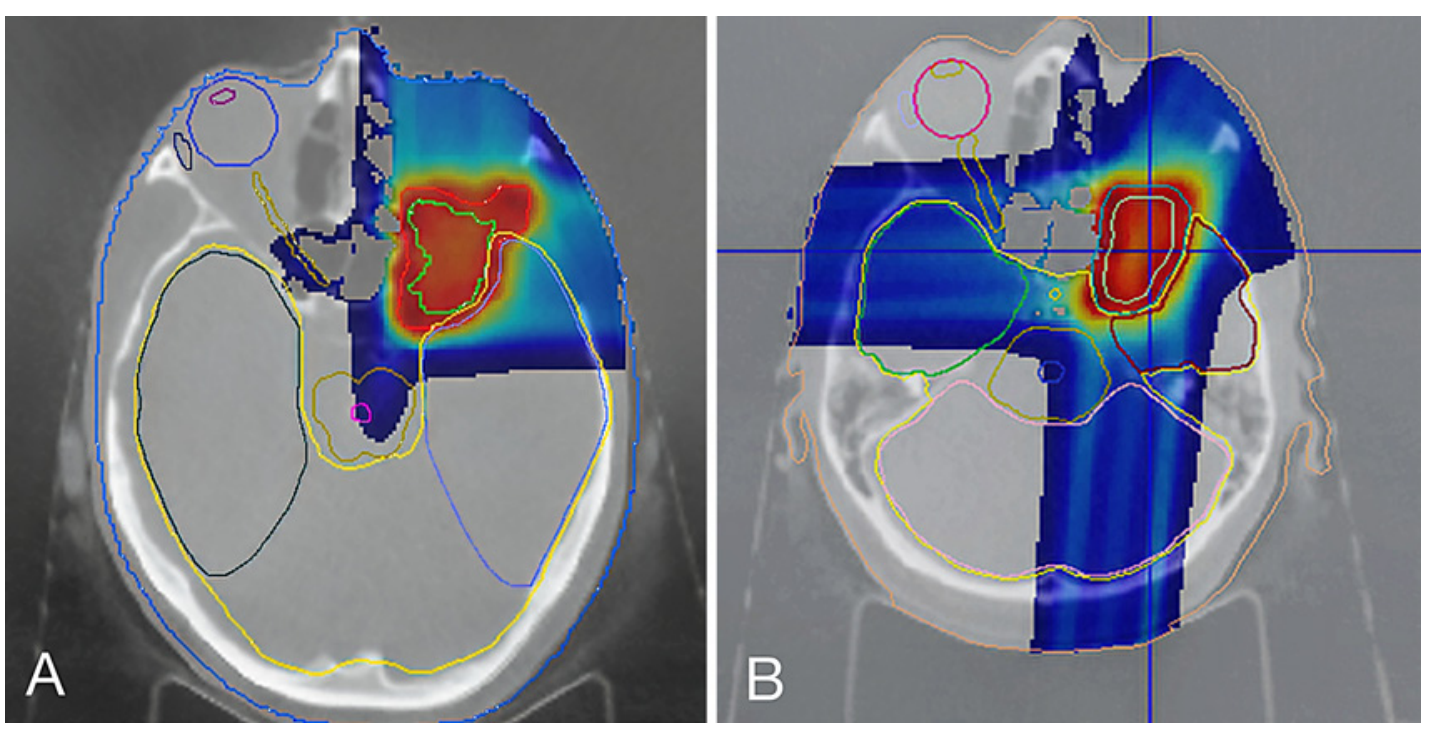

Fig. 2. Dose distribution of the PT courses.

zumab (BVZ), which is known for curing radiation necrosis, was administered as a treatment [11]. In total, the patient received 12 cycles of BVZ, after which the necrosis was finally reported as successfully cured.

In August 2019, another relapse at the same location was reported without signs of brain damage (shown in Fig. 1B). We proposed a combined treatment strategy for the patient: PT with BVZ $5 \mathrm{mg} / \mathrm{kg}$ every 2 weeks concurrently. The 5 th course of radiation therapy was done in August to September 2019. We chose a total dose of 54.0 GyRBE in 30 Fx. The main planning goals were high conformity and normal tissue sparing. The GTV was delineated with MRI and PET co-registration. The final PTV was $12.9 \mathrm{~cm}^{3}$.

The maximum dose to the PTV was $112.2 \%$, and the minimum dose was $86.0 \%$. The $\mathrm{V}_{95}$ was $94.4 \%$, and $D_{95}$ was 53.7 GyRBE. The target coverage was compromised to reduce the dose to brain tissue (temporal lobe). The right eyeball and right lens again received absolutely no dose. The left temporal lobe got 53.7 GyRBE (maximum dose), and the dose given to the opposite temporal lobe was $12.8 \mathrm{GyRBE}$ (maximum dose). The maximum brainstem dose was 21.2 GyRBE, the maximum chiasma dose was $0.5 \mathrm{GyRBE}$, and the maximum dose to the right visual nerve was 14.3 GyRBE. The mean dose to the hypophysis was 14.9 GyRBE, and the midbrain dose was 1.3 GyRBE. Compared to our first PT plan, doses to organs at risk were higher, but this is the result of the field's overlap-sparing strategy. The results of the dosimetry are shown in Figure 2B.

After the treatment, 9 months later, another follow-up MRI (June 2020) showed promising results with tumor shrinkage to $0.4 \mathrm{~cm}^{3}$, without evidence of radionecrosis or any other adverse effects (shown in Fig. 1C).

In August 2020, 1 year after the last treatment, the patient unexpectedly died. The emergency CT scans (shown in Fig. 1D) showed a brain abscess in the left hemisphere, confirmed later by the pathology report. Officially, the adverse event was not related to the irradiation, as it has never been associated with post-RT brain toxicity in the literature. Nevertheless, we can assume that this situation is an indirect treatment side effect. The patient had persistent chronic sinusitis, and the skull base bones exceeded their dose tolerance, which could potentially be open gates to the brain infection. 
Table 1. Cumulative BED to the organs at risk $(\alpha / \beta=3)$

\begin{tabular}{lllll}
\hline & $\begin{array}{l}\text { Optic nerve R } \\
(\text { Dmax })\end{array}$ & $\begin{array}{l}\text { Chiasma } \\
\text { (Dmax) }\end{array}$ & $\begin{array}{l}\text { Temporal lobe L } \\
\text { (Dmax) }\end{array}$ & $\begin{array}{l}\text { Whole brain } \\
\text { (Dmean) }\end{array}$ \\
\hline $1999\left(^{60} \mathrm{Co}, 60 \mathrm{~Gy}\right)$ & 30.4 & 73.6 & 107.1 & 10.3 \\
2009 (SRT, 24 Gy) & 10.0 & 24.6 & 99.8 & 1.7 \\
2013 (SRT, 30 Gy) & 11.3 & 29.1 & 85.3 & 1.3 \\
2016 (PT, 60 GyRBE) & 9.9 & 40.0 & 93.1 & 3.1 \\
2019 (PT, 54 GyRBE) & 17.5 & 3.1 & 86.4 & 3.1 \\
\hline Total BED & 79.1 & 170.4 & 534.7 & 19.5 \\
\hline
\end{tabular}

The patient worked as a nurse until her death and did not demonstrate significant neurocognitive dysfunction. As shown in Table 1, the cumulative doses to the organs at risk were exceeded.

\section{Discussion}

HCP is a rare vascular tumor with a relatively good prognosis. Surgery must be as radical as reasonably achievable to avoid incomplete resection and a high incidence of relapse, together with increased disease duration and a higher risk of malignant development. For anatomical reasons, complete resection is mostly unachievable in an orbital location, so most of the patients are treated with surgery and adjuvant radiation therapy. The issue of HPCs is their mostly local recurrence. Since most recurrent orbital HPCs had already been irradiated, treatment options are usually restricted to repeated incomplete resection, with some additional approaches such as intra-arterial embolization or brachytherapy. Nonetheless, the possibility of re-irradiation for orbital HPCs has not been described at all.

A growing interest in repeated irradiation can be explained by novel approaches to radiation techniques, such as intensity-modulated RT or PT. It is still presented in a small number of studies, with a range of uncertainties such as optimal timing, target volume, fractionation, doses to the recurrent tumor, and organs at risk. These publications are mostly dedicated to the re-irradiation of gliomas with photons or protons, brain metastases, and radiosurgery. It is well known that modern PT achieves maximal dose-to-normal tissue sparing with toxicity benefits compared to photon linear accelerators in varying clinical situations. Galle et al. [13] presented a study in 2015 including 20 patients and showed good tolerance to proton re-irradiation. In 2020 Saeed et al. [14] described 45 patients who underwent proton re-irradiation of glioblastomas, of whom only 4 patients had late toxicity. In 2018 Nieder et al. [15] published a literature review of brain metastasis re-irradiation, which included eight studies involving 1-22 patients. Based on this analysis, the authors considered repeated SRS or SRT (2 or 3 times) after the whole-brain RT as an admissible salvage treatment for individual cases, although with an acceptable risk of brain necrosis. To our knowledge, cases of RT delivered more than 3 times to the same location have not yet been reported. In this extraordinary case, PT helped us achieve adequate tumor control and an acceptable brain toxicity rate, even though the cumulative doses to the organs at risk were maxed out already. Radiation-induced necrosis was observed once and treated with BVZ. Even though we consider re-irradiations as an aggravating factor of chronic sinusitis and death, we believe the provocative treatment strategy of repeating RT was efficient enough and helped to prolong the patient's life for many years.

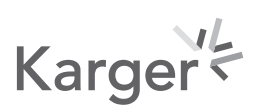




\section{Conclusion}

In a limited number of patients with small target volumes, who have run out of other options, repeated RT can be a treatment of choice with a manageable risk of adverse events. From our experience, with its physical and radiobiological advantages, PT may be the optimal way of providing re-irradiation. The treatment strategy of re-irradiations $>2$ times should be discussed by a multidisciplinary Tumor Board.

\section{Statement of Ethics}

The treatment strategy in this case was approved by the local Ethics Committee. The accompanying manuscript does not contain any studies carried out by the authors on humans or animals.

Written informed consent was obtained from the patient for publication of this case report and any accompanying images, before the patient died.

\section{Conflict of Interest Statement}

All authors know of no competing of interest associated with this publication and have no conflicts of interest to declare.

\section{Funding Sources}

None.

\section{Author Contributions}

The manuscript was read and approved for submission by all named authors. K.G. and I.G. conceived and planned the case report. K.G., A.S., O.G., D.G., O.L., K.M., S.K., and S.D. carried out the treatment and planning. K.G. and I.G. contributed to the interpretation of the results. K.G. and I.G. took the lead in writing the manuscript. S.I. and A.K. made the final approval. All authors provided critical feedback and helped shape the research, analysis, and manuscript.

\section{Availability of Data}

The data used to support the findings of this study are restricted by the Ethical Committee of A.Tsyb MRRC in order to protect the patient's privacy. Data are available upon request from the corresponding author for researchers, who meet the criteria for access to confidential data.

\section{Karger'k}




\section{References}

1 Zimmermann KW. Der feinere Bau der Blutcapillaren. Z Anat Entwickl Gesch. 1923;68(1):29-109.

2 Pacheco LF, Fernandes BF, Miyamoto C, Maloney SC, Arthurs B, Burnier MN Jr. Rapid growth of an orbital hemangiopericytoma with atypical histopathological findings. Clin Ophthalmol. 2014;8:31-3.

3 Mira JG, Chu FC, Fortner JG. The role of radiotherapy in the management of malignant hemangiopericytoma: report of eleven new cases and review of the literature. Cancer. 1977;39(3):1254-9.

4 Tijl JW, Koornneef L, Blank LE. Recurrent hemangiopericytoma and brachytherapy. Doc Ophthalmol. 1992; 82(1-2):103-7.

5 Setzkorn RK, Lee DJ, Iliff NT, Green WR. Hemangiopericytoma of the orbit treated with conservative surgery and radiotherapy. Arch Ophthalmol. 1987;105(8):1103-5.

6 Shinder R, Jackson TL, Araujo D, Prieto VG, Guadagnolo BA, Esmaeli B. Preoperative radiation therapy in the management of recurrent orbital hemangiopericytoma. Ophthal Plast Reconstr Surg. 2011;27(5):e126-8.

7 Mayer R, Sminia P. Reirradiation tolerance of the human brain. Int J Radiat Oncol Biol Phys. 2008;70(5):135060.

8 Krauze AV, Attia A, Braunstein S, Chan M, Combs S, Fietkau R, et al. Expert consensus on re-irradiation for recurrent glioma [published correction in Radiat Oncol. 2018 Jan 18;13(1):8]. Radiat Oncol. 2017;12(1):194.

9 Mizumoto M, Okumura T, Ishikawa E, Yamamoto T, Takano S, Matsumura A, et al. Reirradiation for recurrent malignant brain tumor with radiotherapy or proton beam therapy. Technical considerations based on experience at a single institution. Strahlenther Onkol. 2013;189(8):656-63.

10 Navarria P, Minniti G, Clerici E, Tomatis S, Pinzi V, Ciammella P, et al. Re-irradiation for recurrent glioma: outcome evaluation, toxicity and prognostic factors assessment. A multicenter study of the Radiation Oncology Italian Association (AIRO). J Neurooncol. 2019;142(1):59-67.

11 Zhuang H, Shi S, Yuan Z, Chang JY. Bevacizumab treatment for radiation brain necrosis: mechanism, efficacy and issues. Mol Cancer. 2019;18(1):21.

12 Gulidov IA, Mardynsky YS, Balakin VE, Galkin VN, Gogolin DV, Gordon KB, et al. [New opportunities for proton therapy in Russia]. Vopr Onkol. 2016;62(5):570-2. Russian.

13 Galle JO, McDonald MW, Simoneaux V, Buchsbaum JC. Reirradiation with Proton Therapy for Recurrent Gliomas. Int J Particle Ther. 2015;2(1):11-8.

14 Saeed AM, Khairnar R, Sharma AM, Larson GL, Tsai HK, Wang CJ, et al. Clinical outcomes in patients with recurrent glioblastoma treated with proton beam therapy reirradiation: analysis of the Multi-Institutional Proton Collaborative Group Registry. Adv Radiat Oncol. 2020;5(5):978-83.

15 Nieder C, Yobuta R, Mannsåker B. Second re-irradiation of brain metastases: a review of studies involving stereotactic radiosurgery. Cureus. 2018;10(12):e3712. 